\title{
Swinging a sword: how microtubules search for their targets
}

\author{
Nenad Pavin • Iva M. Tolić-Nørrelykke
}

Received: 7 December 2013/ Accepted: 4 February 2014/Published online: 16 February 2014

(C) The Author(s) 2014. This article is published with open access at Springerlink.com

\begin{abstract}
The cell interior is in constant movement, which is to a large extent determined by microtubules, thin and long filaments that permeate the cytoplasm. To move large objects, microtubules need to connect them to the site of their destination. For example, during cell division, microtubules connect chromosomes with the spindle poles via kinetochores, protein complexes on the chromosomes. A general question is how microtubules, while being bound to one structure, find the target that needs to be connected to this structure. Here we review the mechanisms of how microtubules search for kinetochores, with emphasis on the recently discovered microtubule feature to explore space by pivoting around the spindle pole. In addition to accelerating the search for kinetochores, pivoting helps the microtubules to search for cortical anchors, as well as to self-organize into parallel arrays and asters to target specific regions of the cell. Thus, microtubule pivoting constitutes a mechanism by which they locate targets in different cellular contexts.
\end{abstract}

Keywords Microtubules - Kinetochores - Mitosis · Search mechanism $\cdot$ Pivoting $\cdot$ Angular movement

N. Pavin ( $\square)$

Department of Physics, Faculty of Science, University of

Zagreb, Bijenička 32, 10000 Zagreb, Croatia

e-mail: npavin@phy.hr

I. M. Tolić-Nørrelykke ( $\square)$

Max Planck Institute of Molecular Cell Biology and Genetics, Pfotenhauerstrasse 108, 01307 Dresden, Germany

e-mail: tolic@mpi-cbg.de

I. M. Tolić-Nørrelykke

Division of Molecular Biology, Ruđer Bošković Institute,

Bijenička 54, 10000 Zagreb, Croatia

\section{Introduction}

A living cell is never at rest; organelles and molecular assemblies constantly move in its interior. A large fraction of these movements depend on microtubules, tiny hollow tubes that are found throughout the cytoplasm. Microtubules serve either as tracks to transport cargo such as organelles, vesicles, proteins and RNA, or as ropes on which motor proteins pull in order to move structures such as the mitotic spindle, centrosome and the nucleus (Pavin and Tolić-Nørrelykke 2013; Franker and Hoogenraad 2013; Tolić-Nørrelykke 2008). In order to perform these functions, microtubules must first target their cargo, or regions in the cell where they can load the cargo, or cortical anchor sites for the motors to pull on them. A key question is how microtubules find their targets.

The most prominent example of microtubule-driven movement is the segregation of chromosomes into the two future daughter cells during cell division. At the onset of division, the cell forms a spindle, an accurate self-constructed micro-machine, in which vital parts are microtubules that connect the spindle poles with the chromosomes, and pull on chromosomes to divide the genetic material between the two nascent daughter cells (Cheeseman and Desai 2008). The attachment of microtubules to chromosomes is mediated by kinetochores, protein complexes on the chromosome.

To understand the mechanisms of how microtubules find kinetochores, one needs to know their properties. Microtubules are dynamic polymers that grow or shrink, where a switch between these states occurs in a stochastic manner. In mitotic animal cells, microtubules nucleate mainly from centrosomes, whereas in plants and meiotic cells that lack centrosomes, microtubules nucleate around chromosomes. A variety of microtubule-associated proteins regulate the 
dynamics and nucleation of microtubules (Akhmanova and Steinmetz 2010; Teixido-Travesa et al. 2012). The kinetochore is a protein complex which is very sticky: when it encounters a microtubule, a stable connection is formed between them (Mitchison and Kirschner 1985). The kinetochore size and composition varies between different cell types, as well as the microtubule properties. Thus, different cell types rely on different mechanisms of kinetochore capture. Several mechanisms have been proposed based on experiments, and some of them have been tested theoretically. Here we review the concepts behind these mechanisms with emphasis on the recently discovered microtubule property to explore the space by pivoting around the spindle pole (Kalinina et al. 2013). Finally, we discuss how pivoting helps microtubules to find various targets in different cellular contexts.

\section{Mechanisms of kinetochore capture}

\section{Search-and-capture}

The pioneering idea termed "search-and-capture" relies on the dynamics of microtubules and their nucleation at centrosomes (Kirschner and Mitchison 1986). As a microtubule grows from the centrosome in an arbitrary direction, it probes the space as it searches for kinetochores. Even though a single microtubule probes only one direction, numerous directions will be explored eventually because numerous microtubules grow from the centrosome (Fig. 1a).

In the first theoretical study that investigated the searchand-capture model, the average capture time was calculated for numerous microtubules searching for a single target at a certain distance (Hill 1985). A similar study has shown that the capture time depends strongly on the distance between the kinetochore and the centrosome, and for a typical distance of $10 \mu \mathrm{m}$ it takes ten(s) of minutes to capture a single kinetochore (Holy and Leibler 1994). In case of a system with multiple kinetochores, the time needed to capture all kinetochores grows logarithmically with the number of kinetochores (Wollman et al. 2005; Gopalakrishnan and Govindan 2011). Thus, the searchand-capture model predicts that in a human cell, which has 46 chromosomes, it would take roughly $100 \mathrm{~min}$ to capture all kinetochores. However, human cells capture their kinetochores within 20 min (Wollman et al. 2005), which is five times faster than the theoretical prediction.

Bias in microtubule dynamics

towards the chromosomes

As the search-and-capture model predicts a slower kinetochore capture kinetics than that measured in various cell types, several mechanisms have been proposed to accelerate the search for kinetochores (Duncan and Wakefield 2011; Mogilner and Craig 2010; O'Connell and Khodjakov 2007). One of the reasons for the slow kinetics of capture in the search-and-capture model is that the majority of the microtubules grow in futile directions, towards empty regions of the cell devoid of chromosomes. If the microtubules would "know" where the chromosomes are, this would speed up their search.

To signal their location in the cell, chromosomes create a spatial gradient based on the GTPase Ran (Clarke and Zhang 2008). When bound to GTP, Ran stabilizes microtubules and promotes spindle assembly in Xenopus laevis egg extracts (Zhang et al. 1999; Ohba et al. 1999; Wilde and Zheng 1999; Carazo-Salas et al. 1999; Kalab et al. 1999). The exchange of GDP for GTP on Ran is promoted by its guanine-nucleotide-exchange factor RCC1 (Bischoff and Ponstingl 1991). As RCC1 is bound to the chromosomes, a high local concentration of Ran-GTP is generated at the chromosomes (Carazo-Salas et al. 1999; Kalab et al. 2002). Ran-GTP can diffuse away from the chromosome before the GTP becomes hydrolyzed into GDP, thereby forming a gradient of Ran-GTP that decreases with distance from the chromosomes.

What aspects of microtubule dynamics does the Ran-GTP gradient affect, leading to a more efficient kinetochore capture? Ran-GTP provides a spatial signal that influences microtubule dynamics at a distance from the chromosome, by increasing microtubule rescue frequency and decreasing catastrophe frequency (Carazo-Salas et al. 2001; Wilde et al. 2001). Thus, microtubules that extend into a region close to a chromosome, where the Ran-GTP concentration is high, continue growing, leading to an asymmetric distribution of microtubules with a bias towards the chromosomes (Fig. 1b) (Carazo-Salas and Karsenti 2003).

A theoretical study has explored a biased search-andcapture in the presence of a spatial gradient around the kinetochore of a stabilizing factor that inhibits microtubule catastrophe, thus microtubules that extend towards the kinetochore grow long enough to reach the kinetochore. This bias in microtubule dynamics speeds up the capture of kinetochores (Wollman et al. 2005). Such a bias might be more important for kinetochore capture and spindle assembly in early embryonic systems than in somatic cells, because the cells in early developmental stages are large and may rely on positional cues for microtubule growth more than the smaller somatic cells.

Nucleation of microtubules at the chromosomes and kinetochores

The search-and-capture model relies on the microtubules nucleated at the centrosome, thus this model is also called 
Fig. 1 Models of kinetochore capture. a Search-and-capture; b bias in microtubule dynamics towards the chromosomes; c nucleation of microtubules at the chromosomes; d nucleation of microtubules at the kinetochores; e nucleation of microtubules at spindle microtubules; $\mathbf{f}$ pivoting of microtubules around the spindle pole; $\mathbf{g}$ pivoting of kinetochorebound microtubules;

h kinetochore movements. Microtubules are depicted as green lines, kinetochores as pink spheres on the chromosomes (gray), and centrosomes as gray spheres. Ran-GTP is represented by a pink gradient in (b), and augmin complexes by small blue spheres in (e). Dashed lines mark microtubule growth (a-e), microtubule pivoting (f, g) and the movement of the chromosome (h). In each panel, structures of interest are intensely colored, whereas the remaining parts of the spindle are pale. (Color figure online)
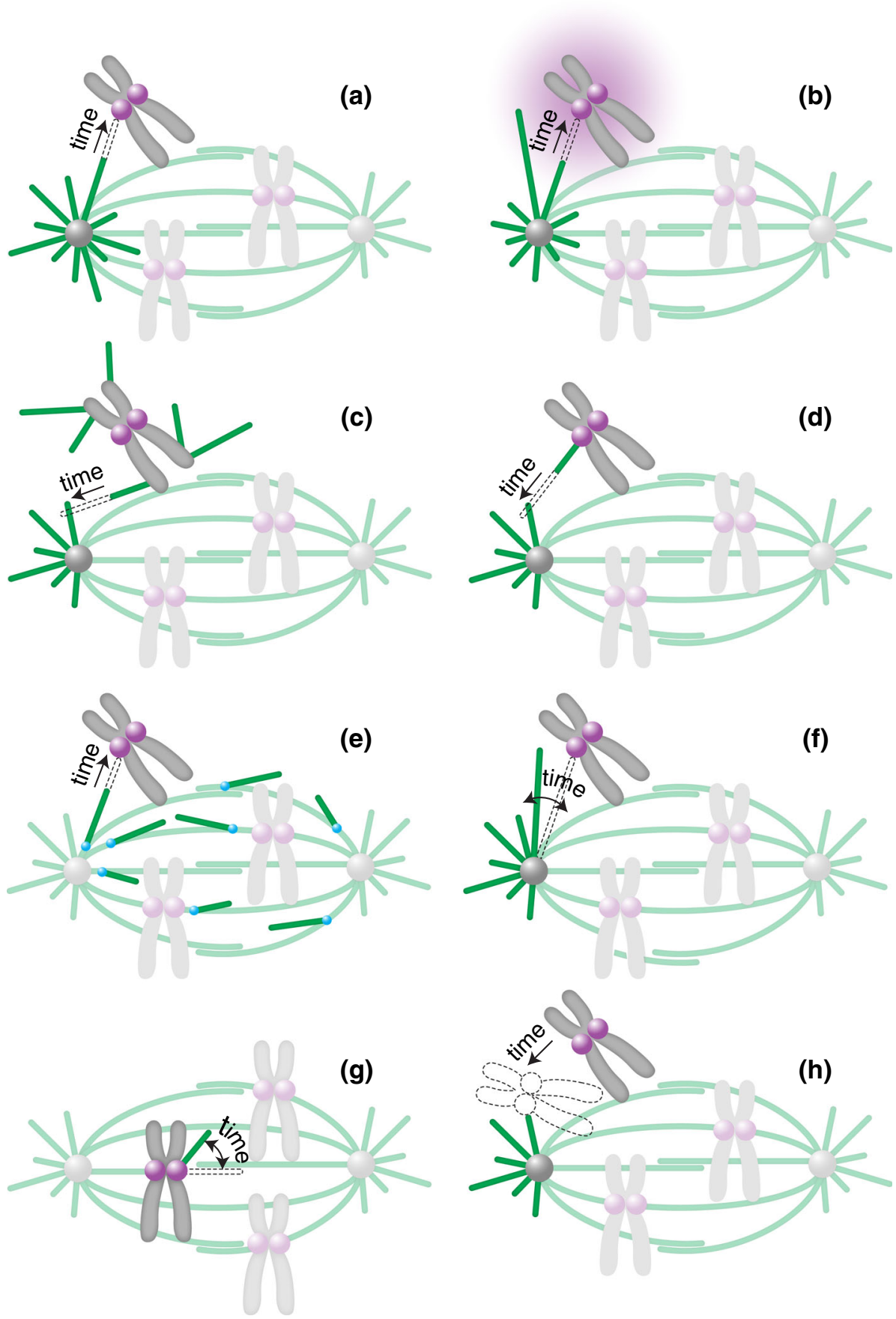

the centrosomal pathway. Yet, microtubules may be nucleated at additional locations. Indeed, it has been shown that chromosomes influence the local assembly of microtubules during metaphase in animal cells, which is known as the chromosomal pathway (Karsenti et al. 1984). Similarly to the stabilization of microtubules in the vicinity of chromosomes, nucleation of microtubules at the chromosomes is also promoted by Ran-GTP (Carazo-Salas et al. 1999). Interactions between chromosomal and centrosomal microtubules may help spindle assembly (Fig. 1c).
Kinetochores are also able to nucleate microtubules, both on isolated mitotic human chromosomes (Telzer et al. 1975) and in mammalian cells in vivo (Witt et al. 1980; De Brabander et al. 1981). Analogously to the microtubules nucleated around the chromosomes, kinetochore-nucleated microtubules may speed up kinetochore capture and spindle assembly (Fig. 1d) (Tulu et al. 2006; Maiato et al. 2004).

On a fully-formed metaphase spindle, kinetochores are attached to the plus ends of microtubules (Euteneuer and 
McIntosh 1981). However, microtubules are generally nucleated from the minus end. What is then the polarity of microtubules that are nucleated at kinetochores? Mammalian and yeast kinetochores have been shown to nucleate microtubules with distal plus ends, just like the centrosomes do (Bergen et al. 1980; Kitamura et al. 2010). Thus, the kinetochores must switch from having proximal minus ends to the proximal plus ends. Upon interaction of kinetochore-nucleated microtubules with the centrosomal ones, the kinetochore microtubules disassemble and the kinetochore remains attached to the centrosomal microtubule. This microtubule has the "correct" polarity with the plus end towards the spindle midzone, allowing the kinetochore to eventually reach the plus end (Kitamura et al. 2010).

The role of microtubules nucleated at kinetochores has been explored in a theoretical study. Computer simulations have shown that the presence of microtubules of a fixed length nucleated at the kinetochores accelerates kinetochore capture, because these microtubules extend the target area that the dynamically unstable centrosomal microtubules must hit (Paul et al. 2009).

\section{Nucleation of microtubules at spindle microtubules}

In addition to microtubule nucleation at the centrosome and the chromosomes, microtubules are nucleated along the pre-existing ones in the spindle (Fig. 1e). These microtubules, which grow parallel or almost parallel to the template microtubule, may participate in the process of kinetochore capture by interacting with a kinetochore, thereby connecting it to the template microtubule. The process of microtubule nucleation throughout the spindle has been observed in cells without and with centrosomes, and it has been shown that it depends on the augmin complex (Burbank et al. 2006; Mahoney et al. 2006; Goshima et al. 2008).

Microtubule pivoting around the spindle pole

A common feature of the mechanisms described above is that microtubules, as they grow, probe the space by their tips. On the contrary, if a microtubule moves laterally it can probe the space by its entire length (Fig. 1f). In this case, the efficiency of the search does not depend on the microtubule growth velocity, but instead on the microtubule length and on how fast the microtubule moves laterally.

In the spindle, where microtubules must link two binding partners, a centrosome and a kinetochore, it is puzzling how a microtubule that is with one end bound to one binding partner can explore the space laterally, as it searches for the other partner. In principle, lateral movement is possible if the bound end of the microtubule is freely jointed to a fixed point, thus allowing the microtubule to pivot around this point. In fission yeast, a microtubule that is bound to the spindle pole body changes its orientation with respect to the cell (Kalinina et al. 2013). In other words, the microtubule pivots around the spindle pole body, swiping through the cell and exploring the space laterally in search for kinetochores, until it approaches and captures a kinetochore. This pivoting movement is random and most likely driven by thermal forces because it does not require ATP. The pivoting can be characterized by an angular diffusion coefficient, which for a $1.5 \mu \mathrm{m}$-long microtubule has a value of $D=0.001 \mathrm{rad}^{2} / \mathrm{s}$. This angular diffusion can explain the measured typical time of kinetochore capture, demonstrating that the search for kinetochores is driven by microtubule pivoting (Kalinina et al. 2013).

Pivoting is not restricted to the microtubules growing from the spindle pole. In Drosophila S2 cells, microtubules extending from the kinetochore have been observed to pivot (Fig. 1g) (Maiato et al. 2004). These kinetochorebound microtubules are initially not oriented towards a spindle pole, but they change their orientation while growing and eventually become captured by the microtubules growing from the centrosome. Similarly, after laser cutting of a microtubule that connects a kinetochore with the centrosome, the microtubule stub that remains associated with the kinetochore pivots as it grows, ultimately becoming integrated into the spindle (Maiato et al. 2004).

\section{Kinetochore movements}

In all the mechanisms described above microtubules probe the space as they search for kinetochores or other microtubules. However, it could be the other way around: by moving around, the kinetochore may probe the space in search for microtubules (Fig. 1h).

The role of kinetochore movements, which include diffusive motion and rotations, in the process of kinetochore capture was studied in human cancer cells (Paul et al. 2009). Experiments have shown that kinetochores, while being associated with the chromosome, perform diffusive movement through the cytoplasm with a diffusion coefficient of $D=0.01 \mu \mathrm{m}^{2} / \mathrm{s}$. The theory, which is based on the measured diffusion of kinetochores and the known properties of microtubules, shows that kinetochore diffusion and rotations accelerate spindle assembly including capture of kinetochores (Paul et al. 2009). Diffusion of kinetochores was measured also in fission yeast, where its value is lower, $D=0.0006 \mu \mathrm{m}^{2} / \mathrm{s}$, and its contribution to the capture process is minor (Kalinina et al. 2013).

As described in this section, numerous mechanisms of kinetochore capture have been identified, but one mechanism does not exclude contributions of others. Thus, a systematic theoretical approach that takes into account all 
mechanisms is needed to understand the process of kinetochore capture. The results depend on the microtubule and kinetochore characteristics included in the model, and thus can differ between various cell types. For example, a single mechanism may be sufficient to explain the kinetics of kinetochore capture in yeast (Kalinina et al. 2013). Alternatively, theory may reveal that the typical capture time can be reproduced only by including several mechanisms, which was shown in a study on human cells (Paul et al. 2009).

\section{Functions of microtubule pivoting in various cellular situations}

Spindle translocation: Pivoting promotes cortical capture of microtubules

During mitosis in budding yeast, the spindle is formed inside the mother cell and has to move through a narrow neck into the daughter cell or the bud, in order to segregate the chromosomes equally between the two cells. Astral microtubules, which grow from the spindle poles, are the main players that move the spindle via their interactions with the cell cortex. Two types of motor proteins are involved in the generation of force on the microtubules and thus on the spindle: myosin $\mathrm{V}$ and cytoplasmic dynein (Yin et al. 2000; Eshel et al. 1993; Li et al. 1993). Myosin V, which is bound to the plus end of the microtubule via the adaptor protein Kar9 (Miller and Rose 1998), walks along the actin cables at the cell cortex, thereby moving the plus end along the cortex. As the actin cables in the mother cell are polarized towards the neck, the plus end eventually reaches the neck. As the pus end is moving, the whole microtubule performs angular movement (Liakopoulos et al. 2003). This movement of the microtubule results in the angular movement of the spindle, which follows the microtubule, becoming oriented towards the neck. Thus, the angular movement of microtubules is important for the orientation of the spindle towards the neck.

Once the spindle is near the neck, astral microtubules enter the daughter cell. To pull the spindle through the neck, dynein that is on the microtubules needs to be offloaded to the cortical anchor protein Num1 (Lee et al. 2005). Angular movement of microtubules, driven by myosin V, most likely helps the microtubules to reach a cortical anchor for dynein (Baumgartner 2011). Thus, the angular movement of microtubules promotes not only the orientation of the spindle towards the neck inside the mother cell, but also the cortical capture of microtubules inside the daughter cell (Fig. 2a), to allow for the interaction with cortical anchors, which is needed for dynein to exert force on the microtubule.
Interphase microtubules: Pivoting helps the formation of a parallel array of microtubule bundles

Microtubule pivoting is important for the generation of self-organized microtubule structures. For example, the rod-shaped fission yeast cells display several microtubule bundles that lie parallel to the long axis of the cell during interphase. Each bundle consists of typically two antiparallel microtubules with their minus-end regions bundled together near the nucleus and the plus ends pointing towards the cell tips (Drummond and Cross 2000). The alignment of the microtubules is crucial for correct positioning of growth sites because the microtubule plus ends deposit polarity factors to the cell tips, thereby restricting the growth pattern of these cells to extension in length at the cell ends (Mata and Nurse 1997; Martin et al. 2005; Tatebe et al. 2005; Baumgartner and Tolić-Nørrelykke 2009). Moreover, the alignment of microtubules during interphase determines the initial alignment of the mitotic spindle (Vogel et al. 2007) and their position determines the position of the nucleus and of the cell division plane (Tolić-Nørrelykke et al. 2005; Daga and Chang 2005; Tolić-Nørrelykke 2010).

How do the microtubule bundles become aligned with the cell axis? Live-cell imaging has shown that microtubules grow from the central region of the cell. Those that contact the cell cortex at an oblique angle, change their angle as they continue growing towards the cell tip and eventually become parallel to the longitudinal axis of the cell (Brunner and Nurse 2000). Thus, through their angular movement, microtubules target specific location in the cell, in this case the cell tips (Fig. 2b). This mechanism of targeting requires regulation of microtubule dynamics, such that the microtubules do not undergo catastrophe too soon after contacting the cell cortex on the lateral sides of the cell, and also not too late after contacting the cortex at the cell tips, which would result in their curling around the cell tips. The regulation of microtubule dynamics is achieved by the proteins that suppress catastrophe, such as the CLIP170 homolog Tip1 (Brunner and Nurse 2000) and the EB-1 homolog Mal3 (Beinhauer et al. 1997).

Microtubule asters in microfabricated chambers:

Pivoting helps microtubules to center the aster

In animal cells, the cleavage plane is determined by the position of the spindle, which in turn is governed by microtubules emanating from the two centrosomes, forming two microtubule asters. Microtubules interact with their plus ends with the cell cortex, where cortical dynein pulls on the microtubule (Gonczy et al. 1999; Grill et al. 2003). In fission yeast, dynein reaches cortical anchor sites by diffusing along the microtubule (Ananthanarayanan et al. 
(a)

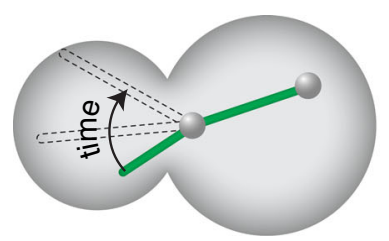

(b)

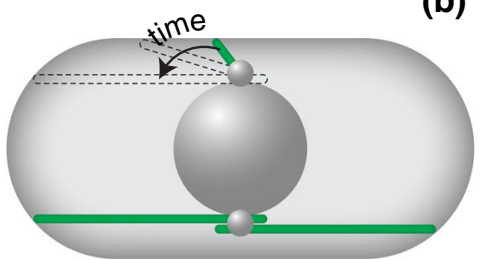

(c)

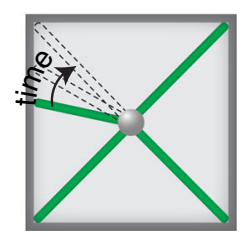

Fig. 2 Microtubule pivoting in various cellular contexts and in vitro. a Pivoting promotes cortical capture of astral microtubules during spindle translocation from the mother to the daughter cell in budding yeast. b Pivoting helps the formation of a parallel array of microtubule bundles in interphase fission yeast cells. $\mathbf{c}$ Pivoting helps microtubules to center the microtubule aster in a microfabricated chamber. Green lines represent microtubules; small gray spheres depict the spindle pole bodies in (a), microtubule-organizing centers in (b) and the centrosome in (c); the large gray sphere in (b) is the nucleus. Dashed lines mark different positions of the microtubules as they pivot and explore the space laterally, searching for their targets. (Color figure online)
2013), and detaches from the cortex in response to load forces (Vogel et al. 2009). An important question is how dyneins, by pulling from the cortex, position the microtubule aster within a confined geometry of the cell.

The process of positioning of microtubule asters can be studied in vitro in a microfabricated chamber. Such approach is ideally suited for investigation of the positioning mechanisms, because one can identify a minimal number of components that are necessary to mimic positioning within cells. A study in which microtubule asters were grown within the chambers with walls coated by dynein motors showed that microtubules are distributed in an anisotropic manner, being preferentially oriented towards the boundaries distal from the centrosome (Laan et al. 2012). Such anisotropy occurs when the tip of the growing microtubule slips along the boundary. As the tip slips, it travels towards the distal boundaries and drives microtubule pivoting that reorients the microtubule towards the distal boundaries (Fig. 2c). Finally, if there is pulling from the cortex, the net force on the anisotropic distribution of microtubules is oriented towards the center of the chamber. Thus, pivoting of the microtubules around the centrosome, as they are becoming reoriented towards the distal boundaries, is crucial for the centering of the centrosome.

\section{Conclusion and outlook}

An important challenge for future work will be to unravel the molecular basis of the free joint that allows for pivoting of the microtubules. Pivoting has been observed in yeast, where the joint is at the spindle pole body, and in higher eukaryotic cells, where microtubules pivot around the centrosome. Although the molecular architecture of the spindle pole body differs from that of the centrosome, the angular movement of the microtubules growing from these two structures seems to be similar. Moreover, microtubules exhibit angular movement also when growing from kinetochores. It will be important to identify the molecules building up the free joint in cases where the microtubule is attached to different cellular structures.

Microtubule pivoting is a major mechanism underlying the search for kinetochores in fission yeast. A combination of experiments and theory has shown that microtubule pivoting speeds up kinetochore capture roughly 30 times compared to a situation in which the microtubules only grow and shrink but do not pivot (Kalinina et al. 2013). It will be interesting to explore to which extent microtubule pivoting contributes to the search for different targets in different cellular settings.

Acknowledgments We thank Matko Glunčić for constructive comments on the manuscript and Ivana Šaric for drawing the figures.

Open Access This article is distributed under the terms of the Creative Commons Attribution License which permits any use, distribution, and reproduction in any medium, provided the original author(s) and the source are credited.

\section{References}

Akhmanova A, Steinmetz MO (2010) Microtubule +TIPs at a glance. J Cell Sci 123(Pt 20):3415-3419

Ananthanarayanan V, Schattat M, Vogel SK, Krull A, Pavin N, TolićNørrelykke IM (2013) Dynein motion switches from diffusive to directed upon cortical anchoring. Cell 153(7):1526-1536

Baumgartner S (2011) Spindle orientation in Saccharomyces cerevisiae. Diploma thesis, Technical University Dresden

Baumgartner S, Tolić-Nørrelykke IM (2009) Growth pattern of single fission yeast cells is bilinear and depends on temperature and DNA synthesis. Biophys J 96(10):4336-4347

Beinhauer JD, Hagan IM, Hegemann JH, Fleig U (1997) Mal3, the fission yeast homologue of the human APC-interacting protein EB-1 is required for microtubule integrity and the maintenance of cell form. J Cell Biol 139(3):717-728

Bergen LG, Kuriyama R, Borisy GG (1980) Polarity of microtubules nucleated by centrosomes and chromosomes of Chinese hamster ovary cells in vitro. J Cell Biol 84(1):151-159

Bischoff FR, Ponstingl H (1991) Catalysis of guanine nucleotide exchange on Ran by the mitotic regulator RCC1. Nature 354(6348):80-82

Brunner D, Nurse P (2000) CLIP170-like tip1p spatially organizes microtubular dynamics in fission yeast. Cell 102(5):695-704 
Burbank KS, Groen AC, Perlman ZE, Fisher DS, Mitchison TJ (2006) A new method reveals microtubule minus ends throughout the meiotic spindle. J Cell Biol 175(3):369-375

Carazo-Salas RE, Karsenti E (2003) Long-range communication between chromatin and microtubules in Xenopus egg extracts. Curr Biol 13(19):1728-1733

Carazo-Salas RE, Guarguaglini G, Gruss OJ, Segref A, Karsenti E, Mattaj IW (1999) Generation of GTP-bound Ran by RCC1 is required for chromatin-induced mitotic spindle formation. Nature 400(6740):178-181

Carazo-Salas RE, Gruss OJ, Mattaj IW, Karsenti E (2001) Ran-GTP coordinates regulation of microtubule nucleation and dynamics during mitotic-spindle assembly. Nat Cell Biol 3(3):228-234

Cheeseman IM, Desai A (2008) Molecular architecture of the kinetochore-microtubule interface. Nat Rev Mol Cell Biol 9(1):33-46

Clarke PR, Zhang C (2008) Spatial and temporal coordination of mitosis by Ran GTPase. Nat Rev Mol Cell Biol 9(6):464-477

Daga RR, Chang F (2005) Dynamic positioning of the fission yeast cell division plane. Proc Natl Acad Sci USA 102(23):8228-8232

De Brabander M, Geuens G, De Mey J, Joniau M (1981) Nucleated assembly of mitotic microtubules in living PTK2 cells after release from nocodazole treatment. Cell Motil 1(4):469-483

Drummond DR, Cross RA (2000) Dynamics of interphase microtubules in Schizosaccharomyces pombe. Curr Biol 10(13):766-775

Duncan T, Wakefield JG (2011) 50 ways to build a spindle: the complexity of microtubule generation during mitosis. Chromosome Res 19(3):321-333

Eshel D, Urrestarazu LA, Vissers S, Jauniaux JC, van Vliet-Reedijk JC, Planta RJ, Gibbons IR (1993) Cytoplasmic dynein is required for normal nuclear segregation in yeast. Proc Natl Acad Sci USA 90(23):11172-11176

Euteneuer U, McIntosh JR (1981) Structural polarity of kinetochore microtubules in PtK1 cells. J Cell Biol 89(2):338-345

Franker MA, Hoogenraad CC (2013) Microtubule-based transportbasic mechanisms, traffic rules and role in neurological pathogenesis. J Cell Sci 126(Pt 11):2319-2329

Gonczy P, Pichler S, Kirkham M, Hyman AA (1999) Cytoplasmic dynein is required for distinct aspects of MTOC positioning, including centrosome separation, in the one cell stage Caenorhabditis elegans embryo. J Cell Biol 147(1):135-150

Gopalakrishnan M, Govindan BS (2011) A first-passage-time theory for search and capture of chromosomes by microtubules in mitosis. Bull Math Biol 73(10):2483-2506

Goshima G, Mayer M, Zhang N, Stuurman N, Vale RD (2008) Augmin: a protein complex required for centrosome-independent microtubule generation within the spindle. J Cell Biol 181(3):421-429

Grill SW, Howard J, Schaffer E, Stelzer EH, Hyman AA (2003) The distribution of active force generators controls mitotic spindle position. Science 301(5632):518-521

Hill TL (1985) Theoretical problems related to the attachment of microtubules to kinetochores. Proc Natl Acad Sci USA 82(13):4404-4408

Holy TE, Leibler S (1994) Dynamic instability of microtubules as an efficient way to search in space. Proc Natl Acad Sci USA 91(12):5682-5685

Kalab P, Pu RT, Dasso M (1999) The ran GTPase regulates mitotic spindle assembly. Curr Biol 9(9):481-484

Kalab P, Weis K, Heald R (2002) Visualization of a Ran-GTP gradient in interphase and mitotic Xenopus egg extracts. Science 295(5564):2452-2456

Kalinina I, Nandi A, Delivani P, Chacon MR, Klemm AH, RamunnoJohnson D, Krull A, Lindner B, Pavin N, Tolić-Nørrelykke IM (2013) Pivoting of microtubules around the spindle pole accelerates kinetochore capture. Nat Cell Biol 15(1):82-87
Karsenti E, Newport J, Kirschner M (1984) Respective roles of centrosomes and chromatin in the conversion of microtubule arrays from interphase to metaphase. J Cell Biol 99(1 Pt 2):47s$54 \mathrm{~s}$

Kirschner M, Mitchison T (1986) Beyond self-assembly: from microtubules to morphogenesis. Cell 45(3):329-342

Kitamura E, Tanaka K, Komoto S, Kitamura Y, Antony C, Tanaka TU (2010) Kinetochores generate microtubules with distal plus ends: their roles and limited lifetime in mitosis. Dev Cell 18(2):248-259

Laan L, Pavin N, Husson J, Romet-Lemonne G, van Duijn M, Lopez MP, Vale RD, Julicher F, Reck-Peterson SL, Dogterom M (2012) Cortical dynein controls microtubule dynamics to generate pulling forces that position microtubule asters. Cell 148(3):502-514

Lee WL, Kaiser MA, Cooper JA (2005) The offloading model for dynein function: differential function of motor subunits. J Cell Biol 168(2):201-207

Li YY, Yeh E, Hays T, Bloom K (1993) Disruption of mitotic spindle orientation in a yeast dynein mutant. Proc Natl Acad Sci USA 90(21): 10096-10100

Liakopoulos D, Kusch J, Grava S, Vogel J, Barral Y (2003) Asymmetric loading of Kar9 onto spindle poles and microtubules ensures proper spindle alignment. Cell 112(4):561-574

Mahoney NM, Goshima G, Douglass AD, Vale RD (2006) Making microtubules and mitotic spindles in cells without functional centrosomes. Curr Biol 16(6):564-569

Maiato H, Rieder CL, Khodjakov A (2004) Kinetochore-driven formation of kinetochore fibers contributes to spindle assembly during animal mitosis. J Cell Biol 167(5):831-840

Martin SG, McDonald WH, Yates JR 3rd, Chang F (2005) Tea4p links microtubule plus ends with the formin for $3 p$ in the establishment of cell polarity. Dev Cell 8(4):479-491

Mata J, Nurse P (1997) teal and the microtubular cytoskeleton are important for generating global spatial order within the fission yeast cell. Cell 89(6):939-949

Miller RK, Rose MD (1998) Kar9p is a novel cortical protein required for cytoplasmic microtubule orientation in yeast. J Cell Biol 140(2):377-390

Mitchison TJ, Kirschner MW (1985) Properties of the kinetochore in vitro. II. Microtubule capture and ATP-dependent translocation. J Cell Biol 101(3):766-777

Mogilner A, Craig E (2010) Towards a quantitative understanding of mitotic spindle assembly and mechanics. J Cell Sci $123(\mathrm{Pt}$ 20):3435-3445

O'Connell CB, Khodjakov AL (2007) Cooperative mechanisms of mitotic spindle formation. J Cell Sci 120(Pt 10):1717-1722

Ohba T, Nakamura M, Nishitani H, Nishimoto T (1999) Selforganization of microtubule asters induced in Xenopus egg extracts by GTP-bound Ran. Science 284(5418):1356-1358

Paul R, Wollman R, Silkworth WT, Nardi IK, Cimini D, Mogilner A (2009) Computer simulations predict that chromosome movements and rotations accelerate mitotic spindle assembly without compromising accuracy. Proc Natl Acad Sci USA 106(37):15708-15713

Pavin N, Tolić-Nørrelykke IM (2013) Dynein, microtubule and cargo: a menage a trois. Biochem Soc Trans 41(6):1731-1735

Tatebe H, Shimada K, Uzawa S, Morigasaki S, Shiozaki K (2005) Wsh3/Tea4 is a novel cell-end factor essential for bipolar distribution of Tea1 and protects cell polarity under environmental stress in S. pombe. Curr Biol 15(11):1006-1015

Teixido-Travesa N, Roig J, Luders J (2012) The where, when and how of microtubule nucleation - one ring to rule them all. J Cell Sci 125(Pt 19):4445-4456

Telzer BR, Moses MJ, Rosenbaum JL (1975) Assembly of microtubules onto kinetochores of isolated mitotic chromosomes of HeLa cells. Proc Natl Acad Sci USA 72(10):4023-4027 
Tolić-Nørrelykke IM (2008) Push-me-pull-you: how microtubules organize the cell interior. Eur Biophys J 37(7):1271-1278

Tolić-Nørrelykke IM (2010) Force and length regulation in the microtubule cytoskeleton: lessons from fission yeast. Curr Opin Cell Biol 22(1):21-28

Tolić-Nørrelykke IM, Sacconi L, Stringari C, Raabe I, Pavone FS (2005) Nuclear and division-plane positioning revealed by optical micromanipulation. Curr Biol 15(13):1212-1216

Tulu US, Fagerstrom C, Ferenz NP, Wadsworth P (2006) Molecular requirements for kinetochore-associated microtubule formation in mammalian cells. Curr Biol 16(5):536-541

Vogel SK, Raabe I, Dereli A, Maghelli N, Tolić-Nørrelykke I (2007) Interphase microtubules determine the initial alignment of the mitotic spindle. Curr Biol 17(5):438-444

Vogel SK, Pavin N, Maghelli N, Julicher F, Tolić-Nørrelykke IM (2009) Self-organization of dynein motors generates meiotic nuclear oscillations. PLoS Biol 7(4):e1000087

Wilde A, Zheng Y (1999) Stimulation of microtubule aster formation and spindle assembly by the small GTPase Ran. Science 284(5418):1359-1362
Wilde A, Lizarraga SB, Zhang L, Wiese C, Gliksman NR, Walczak CE, Zheng Y (2001) Ran stimulates spindle assembly by altering microtubule dynamics and the balance of motor activities. Nat Cell Biol 3(3):221-227

Witt PL, Ris H, Borisy GG (1980) Origin of kinetochore microtubules in Chinese hamster ovary cells. Chromosoma 81(3):483-505

Wollman R, Cytrynbaum EN, Jones JT, Meyer T, Scholey JM, Mogilner A (2005) Efficient chromosome capture requires a bias in the 'search-and-capture' process during mitotic-spindle assembly. Curr Biol 15(9):828-832

Yin H, Pruyne D, Huffaker TC, Bretscher A (2000) Myosin V orientates the mitotic spindle in yeast. Nature 406(6799):1013-1015

Zhang C, Hughes M, Clarke PR (1999) Ran-GTP stabilises microtubule asters and inhibits nuclear assembly in Xenopus egg extracts. J Cell Sci 112(Pt 14):2453-2461 